\title{
A Radiation Analysis of 500W RTPV System having Cylindrical Sources
}

\author{
Seong Jae Cheon ${ }^{1}$, Ser Gi Hong ${ }^{1, *}$ Young Suk Nam ${ }^{2}$ and Jung Hun Lee ${ }^{2}$ \\ ${ }^{1}$ Dept. of Nuclear Engineering, Kyung Hee University, 1732 Deokyoungdaero, Giheung-gu, Yongin, Gyeonggi-do 446-701, Republic of \\ korea \\ ${ }^{2}$ Dept. of Mechanical Engineering, Kyung Hee University, 1732 Deokyoungdaero, Giheung-gu, Yongin, Gyeonggi-do 446-701, Republic \\ of korea
}

\begin{abstract}
In this work, the radiation shielding analysis for a RTPV (Radioisotope Thermo-Photo-Voltaic) device using ${ }^{238} \mathrm{PuO}_{2}$ was performed to estimate the radiation dose distribution over source region and radiation doses in a specified tally zone. The radiation source intensities and spectra were evaluated with ORIGEN-S and the detailed shielding analyses were performed with MCNP6 and MONACO/MAVRIC (in SCALE 6.1). In particular, comparative shielding analysis using two different types of RTPV (cubic homogeneous source type and cylindrical heterogeneous source type) were performed to show their relative performances. The results show that the RTPV device using the heterogeneous cylindrical source type has lower dose values by $5 \sim 11 \%$ depending on the packing factor at the measurement cell than the one using the homogeneous cubic source type. The additional thermal analysis using COMSOL shows that the temperature distribution of the heterogeneous cylindrical source type is more flat than that of a homogeneous cubic source type.
\end{abstract}

\section{INTRODUCTION}

Radioisotope Thermoelectric Generators (RTG) has been considered as a power supply system for long-lived operation in space. RTG is an essentially nuclear battery that converts the heat resulted from the radioactive decay into electricity. RTG has the advantage of high energy density and long life operation due to very long halflives of the radioisotopes. However, the RTG systems have relatively low thermoelectric energy conversion efficiency of 3-7\% because RTGs rely on the thermoscouples to convert heat to electricity. On the other hand, in a RTPV, radiations such as beta or alpha particles heat an emitter material which emits photon to a PV cell converting the photons into electricity. Therefore, a RTPV (Radioisotope Thermo-Photo-Voltaic) system has typically much higher energy conversion efficiency of $15 \sim 30 \%$ than RTG system [1].

Recently, we have worked on a project for designing a small RTPV device $(500 \mathrm{~W})$ and simulating its thermal performances by using a small scale experiment. In this project, the kinetic energy of alpha particles $(\sim 5 \mathrm{MeV})$ from ${ }^{238} \mathrm{PuO}_{2}$ is considered as a long-term power supplier for the RTPV device. The alpha decay of ${ }^{238} \mathrm{Pu}$ accompanies additional neutrons by $(\alpha, n)$ reactions of ${ }^{17} \mathrm{O}$ and ${ }^{18} \mathrm{O}$, and also the spontaneous fission of ${ }^{238} \mathrm{Pu}$ generates neutrons. In addition to these neutrons, these radioactive decay and reactions are accompanied by gammas. Therefore the shielding of these radiations is very important for radiation safety.
The objective of this work is to perform radiation dose analysis of heat source region for the RTPV system. In particular, a comparative shielding analysis using two different types of RTPV (cubic homogeneous source type and cylindrical heterogeneous source type) was performed to show their relative performances. In addition, we estimated the temperature distribution for thermal safety analysis of RTPV devices.

\section{RADIATION SAFETY ANALYSIS}

\subsection{Radiation shielding analysis procedure}

Fig. 1 shows the calculation procedure for the safety analysis of RTPV system. The radiation safety analysis consists of two steps. First, the ORIGEN-S code was used to evaluate the intensities and spectra of neutrons and gamma rays emitted from the ${ }^{238} \mathrm{PuO}_{2}$ [2]. Then, the doses are estimated by performing neutron and gamma transport simulations using two Monte Carlo codes and the source intensities and spectra estimated with ORIGEN-S. In this work, we used MCNP6 and MONACO/MAVRIC to perform the neutron and gamma transport calculations $[3,4]$.

\footnotetext{
* Corresponding author: sergihong@khu.ac.kr
} 


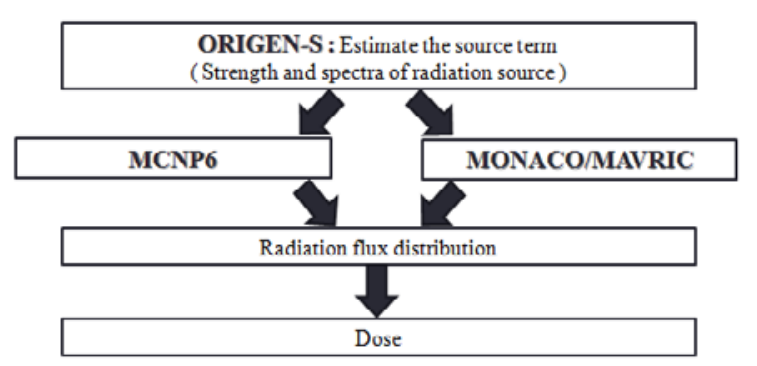

Fig. 1. The calculation procedure for a safety analysis.

\subsection{Source term evaluation with ORIGEN-S}

ORIGEN-S which is a part of SCALE6.1 is a computer code for evaluating the radioactive decay of radionuclide and the amount of nuclides after the neutron irradiation and cooling [2]. In this work, we used ORIGEN-S code for evaluating the intensities and spectra emitted from the source of RTPV system. The radiation source terms should be described prior to the detailed radiation safety analysis and their accuracies are quite important for accurate estimation of radiation dose.

The alpha decay of ${ }^{238} \mathrm{Pu}$ releases $\sim 5.5 \mathrm{MeV}$ energy and also additional neutrons are emitted as result of the reactions (i.e., $(\alpha, n)$ reactions) of ${ }^{17} \mathrm{O}$ and ${ }^{18} \mathrm{O}$ with the alpha particles. In addition, the spontaneous fission of ${ }^{238} \mathrm{Pu}$ generates neutrons. Table 1 summarizes the neutron source intensities estimated using ORIGEN-S for a $500 \mathrm{~W}$ heat generation.

Table 1. Neutron source intensities (neutrons/sec) for $500 \mathrm{~W}$ ${ }^{238} \mathrm{PuO}_{2}$ source.

\begin{tabular}{lc}
\hline \multicolumn{1}{c}{ Components } & Values \\
\hline Spontaneous fission & $2.39 \times 10^{6}$ \\
\hline Delayed neutrons & Negligible \\
\hline$(\alpha, \mathrm{n})$ reactions $-\left(\right.$ mainly from $\left.{ }^{18} \mathrm{O}\right)$ & $1.253 \times 10^{7}$ \\
\hline$(\alpha, \mathrm{n})$ reactions $-\left(\right.$ from $\left.{ }^{17} \mathrm{O}\right)$ & $9.996 \times 10^{5}$ \\
\hline Total $(\alpha, \mathrm{n})$ reactions & $1.253 \times 10^{7}$ \\
\hline Total & $1.492 \times 10^{7}$ \\
\hline
\end{tabular}

For gamma source, the most dominant contribution is from the radioactive decay of ${ }^{238} \mathrm{Pu}$ while the spontaneous fission of ${ }^{238} \mathrm{Pu}$ and $(\alpha, \mathrm{n})$ reactions generate additional gammas [5]. The gammas from the decay of ${ }^{238} \mathrm{Pu}$ have peaks at $43 \mathrm{keV}, 99 \mathrm{keV}$, and $152 \mathrm{keV}$ energies These spectra are obtained by using ORIGEN-S with the 200 and 47 energy group structures for neutron and gamma, respectively.

\subsection{Radiation safety analysis with MCNP6 and MONACO/MAVRIC (SCALE6.1)}

\subsubsection{Computer codes for radiation dose analysis}

We performed radiation safety analysis by using MCNP6 and MONACO/MAVRIC codes with the specified intensities and spectra of the radiation source determined with ORIGEN-S. MCNP6 is an advanced general purpose (continuous-energy and general geometry) simulation code that transports 37 different particles for criticality, shielding, dosimetry and so on. It is the latest version of the famous MCNP code [4]. MONACO is a new 3-D Monte Carlo code being developed within SCALE for shielding calculations. It is a multi-group Monte Carlo transport code with fixed sources for shielding applications. The tallies available in MONACO include point detectors, region-based flux tallies and mesh tallies. Specially, mesh tally values can be viewed with the mesh file viewer in SCALE6.1. The MAVRIC sequence is completely automated for shielding analysis [3].

\subsubsection{Description of RTPV using homogeneous cubic source}

In our previous work, a homogeneous cubic source sequentially surrounded by a tungsten material region and a thin emitter region of tantalum was considered for RTPV device [6]. In this work, the effects of the packing factor which is the ratio of heat source region (i.e., ${ }^{238} \mathrm{PuO}_{2}$ region) volume to the total region enclosed by the emitter on the radiation dose are analysed. In particular, a cladding is considered to enclose the source region in order to prevent releasing of ${ }^{238} \mathrm{PuO}_{2}$ under accident. The size of the source is fixed because the release of heat source is fixed to $500 \mathrm{~W}$. The total region enclosed by emitter consists of a $4.4910 \mathrm{~cm} \mathrm{x} 4.4910 \mathrm{~cm}$ $\mathrm{x} 4.4910 \mathrm{~cm}$ central cubic source region and its three surrounding regions. The central source region is first surrounded by an iridium (Ir) cladding region. The second surrounding region is a tungsten (W) radiation shielding region that is followed by a $0.5 \mathrm{~cm}$ thick tantalum (Ta) outer emitter region. The thickness of the shielding material is determined by the packing factor. Fig. 2 shows the configuration of the cubic heat source region for RTPV device.

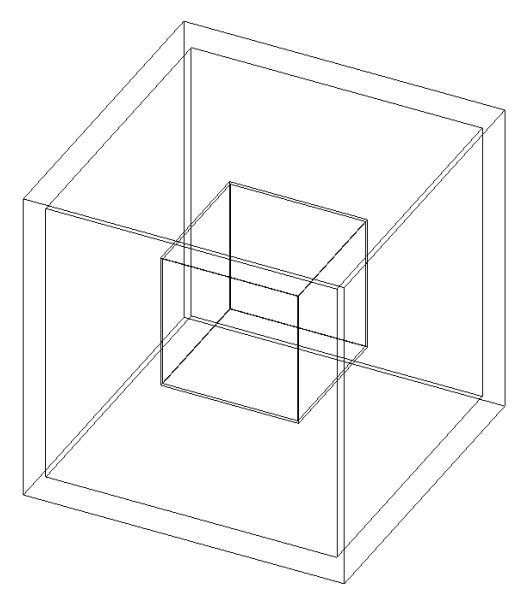

Fig. 2. Geometric model for the source of a cubic design 


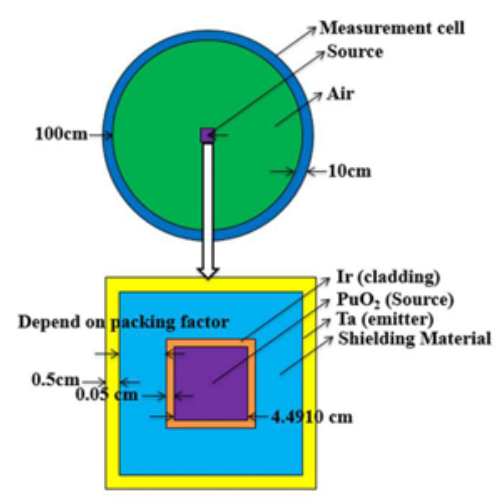

Fig. 3. Geometric model for the RTPV device of the cubic source design

The radiation dose is estimated in a $10 \mathrm{~cm}$ thick spherical measurement shell which is located at $100 \mathrm{~cm}$ distance from the center of the source. The region between RTPV device and the measurement spherical shell is assumed to be filled with air. Fig. 3 shows MCNP6 and MONACO/MAVRIC geometric modelling for radiation safety analysis. The radiation dose was converted from the radiation fluxes using the ICRU-57 and the ICRP-21 dose conversion factors for SCALE6.1 [7] and MCNP6 [4], respectively.

\subsubsection{Description of RTPV using cylindrical sources}

In this work, we also considered RTPV device where four separate cylindrical source zones having iridium cladding are symmetrically located and the dose values in a $10 \mathrm{~cm}$ thick spherical measurement shell which is located at $100 \mathrm{~cm}$ distance from the center of the source are estimated with MONACO/MAVRIC. In particular, the dose values for this RTPV device having cylindrical sources are compared with those for the one having cubic source. The reason for the consideration of separated cylindrical sources is to flatten the neutron flux and temperature distributions. As in the previous design using a single cubic source, the total volume of the source is fixed and it is determined to generate $500 \mathrm{~W}$ heat. Fig. 4 shows the geometric model of the RTPV device having separated cylindrical sources for radiation safety analysis. A cylinder source whose radius and height are $1.217 \mathrm{~cm}$ and $4.867 \mathrm{~cm}$, respectively releases $125 \mathrm{~W}$ heat.

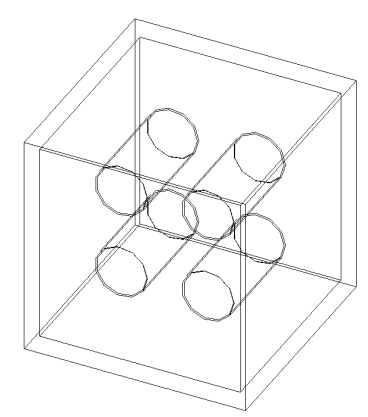

Fig. 4. Geometric model for the source of the cylindrical design
Fig. 5 shows MCNP6 and MONACO/MAVRIC geometric model for the RTPV device of the cylindrical source design. The distances between the cylindrical sources were adjusted to keep the same distance between the outer surface of the source cladding and the inner surface of the emitter for different packing factors.

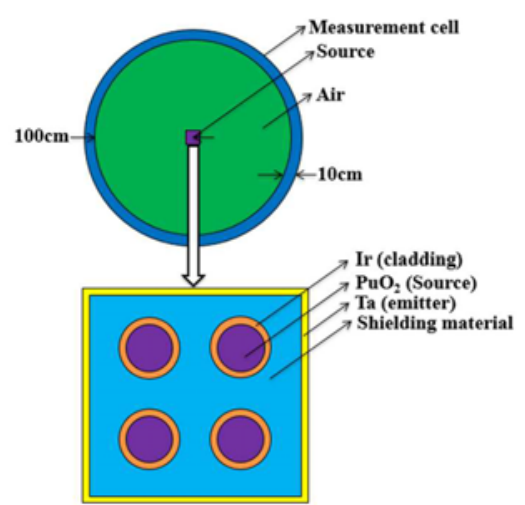

Fig. 5. Geometric model for the RTPV device of the cylindrical source design

\subsubsection{Evaluation of the radiation doses}

The thermal-to-electric energy conversion efficiency of RTPV strongly depends on the packing factor and the increase of packing factor usually leads to the higher conversion efficiency. On the other hand, the effect of the packing factor on the radiation dose is also needed to be estimated. In this work, the effects of the packing factor on the radiation dose are comparatively estimated for the RTPV devices having a single cubic source and separated cylindrical sources described above. Table 2 compares the total radiation doses in a $10 \mathrm{~cm}$ thick measurement shell that is located at $100 \mathrm{~cm}$ distance from the center of the source for the RTPV devices having a single cubic source and the separated cylindrical sources. As shown in Table 2, the dose values for the separated cylindrical sources are smaller than that for the single cubic sources for all the packing factors. In particular, it is noted that the difference in the dose values between these two different source configurations has the largest value of $\sim 11.1 \%$ for the smallest packing factor (i.e., $10 \%)$. As expected, these differences in the dose values decrease as the packing factor increase. Fig. 6 compares these dose values graphically.

Table 2. Doses in the measurement sell (mSv/hr)

Packing factor (\%) Total dose value

\begin{tabular}{ccc}
\hline & Cubic source & Cylindrical sources \\
\hline 10 & $1.79 \mathrm{E}-01$ & $1.59 \mathrm{E}-01\left(11.1^{\mathrm{a}}\right)$ \\
\hline 30 & $1.94 \mathrm{E}-01$ & $1.78 \mathrm{E}-01(8.2)$ \\
\hline 50 & $1.99 \mathrm{E}-01$ & $1.87 \mathrm{E}-01(6.2)$ \\
\hline 55 & $1.93 \mathrm{E}-01$ & $1.89 \mathrm{E}-01(5.3)$ \\
\hline
\end{tabular}

${ }^{a}$ Difference (\%) between the dose values for the different source configurations 


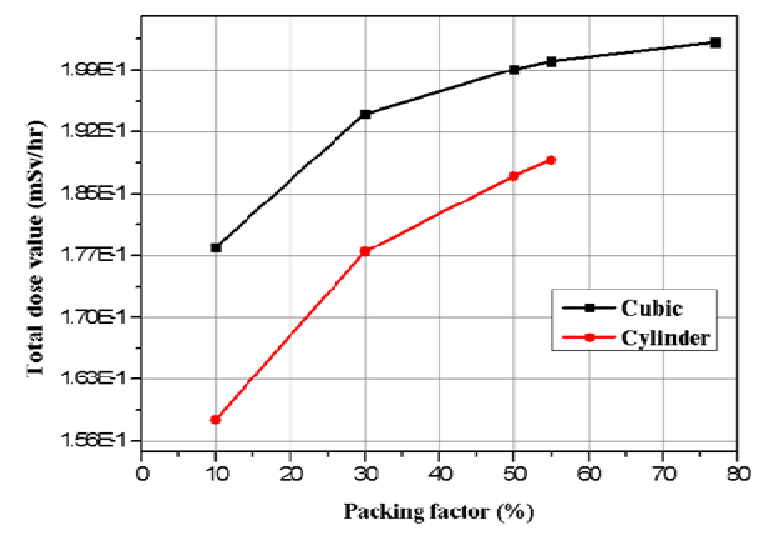

Fig. 6. Comparison of the total doses for a 500W heat generation between two source designs

Actually, the neutron dose is much more dominant than the gamma dose. Figures 7(a) and 7(b) compare the neutron dose distributions at the center planes for the RTPV devices having a cubic source and separated cylindrical sources, respectively. As expected, it is shown that the neutron dose distribution for the separated cylindrical sources is much more flat and the dose at outer surface for this case is lower than those for the single cubic source.
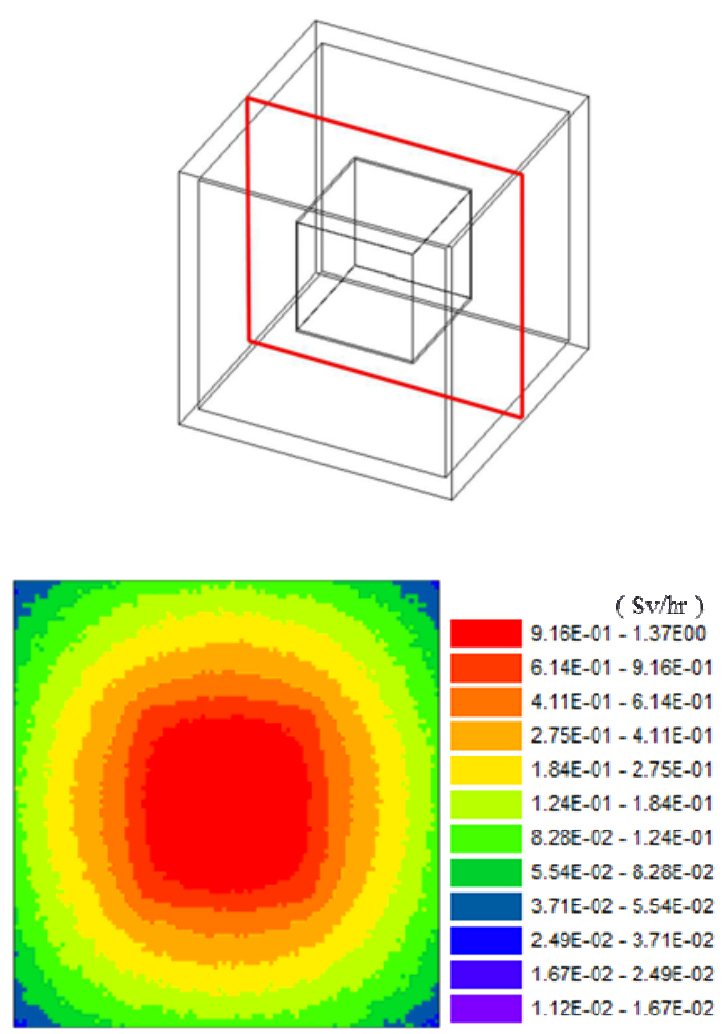

(a) A cubic source design
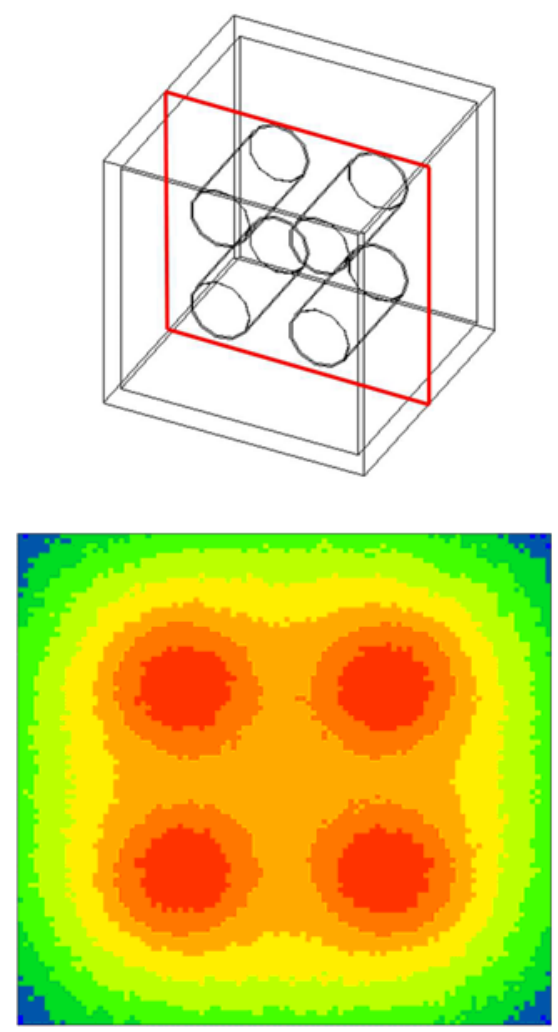

(b) Cylindrical sources design

Fig. 7. Comparison of the neutron dose distributions at the central planes (with same scale in dose, $\mathrm{Sv} / \mathrm{hr}$ )

\section{Thermal analysis}

\subsection{Modelling with COMSOL for thermal analysis}

Next, we performed thermal safety analysis by using the COMSOL program. The COMSOL Multiphysics program is a powerful interactive environment for modeling and solving all kinds of scientific and engineering problems based on partial differential equations [8]. We analyzed temperature distributions using COMSOL and tetrahedral meshes. The geometrical modelings for the safety analysis are same as those for the radiation safety analysis. Table 3 shows the input physical parameters for thermal analysis of the RTPV devices.

Table 3. Input parameters for thermal analysis for the RTPV devices

\begin{tabular}{ccccc}
\hline Parameter & PuO $_{2}$ & Ir & W & Ta \\
\hline $\begin{array}{c}\text { Thermal conductivity } \\
(\mathrm{W} / \mathrm{m} \cdot \mathrm{K})\end{array}$ & 6.3 & 147 & 173 & 57.5 \\
\hline $\begin{array}{c}\text { Heat capacity } \\
(\mathrm{J} / \mathrm{kg} \cdot \mathrm{K})\end{array}$ & 240 & 233 & 130 & 140 \\
\hline Density $\left(\mathrm{g} / \mathrm{cm}^{3}\right)$ & 11.5 & 22.42 & 19.25 & 16.69 \\
\hline
\end{tabular}


Eq. (1) shows the radiation boundary condition for estimating temperature distribution for the RTPV devices.

$$
Q=\varepsilon \sigma T^{4}
$$

where $Q$ is the outward heat flux $\left(\mathrm{W} / \mathrm{m}^{2}\right), \varepsilon$ is the emissivity, $\sigma$ is the Stefan-Boltzmann constant $(5.67 \mathrm{x}$ $10^{-8}$ ) and $T$ is temperature $(\mathrm{K})$. Figure 8 shows the emissivity as a function of temperature which is from the experiment data for the RTPV device.

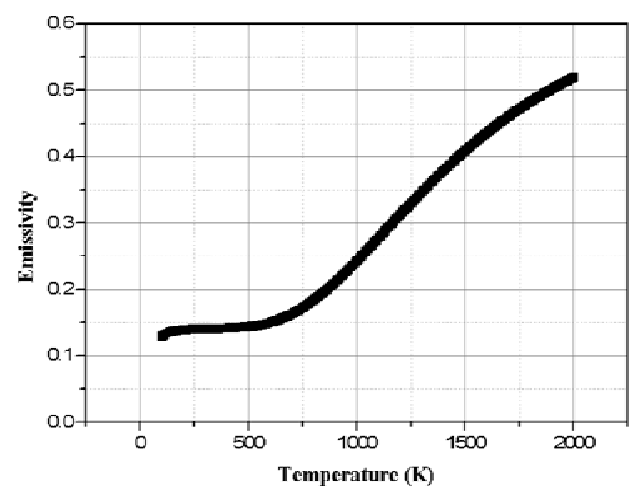

Fig. 8. Emissivity $(\varepsilon)$ by temperature from the experiment data

\subsection{Result of the thermal analysis}

Figures 9(a) and 9(b) compare the temperature distributions at the axially central planes for the different source designs. Figure 9 shows the temperature distribution for the cylindrical sources design is much more flat than that for the cubic source design. Table 4 shows the maximum temperatures and the average temperatures on the outer faces for the two RTPVs having different source shapes. For the RTPV having cubic source, the maximum temperature is about $993.4 \mathrm{~K}$ which is higher by $\sim 69 \mathrm{~K}$ than that of the one having the four cylindrical sources while the average temperatures on their outer faces are almost the same. Therefore, the temperature gradients for the cylindrical sources are smaller than that those for the cubic source design, which means that the cylindrical sources design will enhance the thermal integrity of the RTPV system.

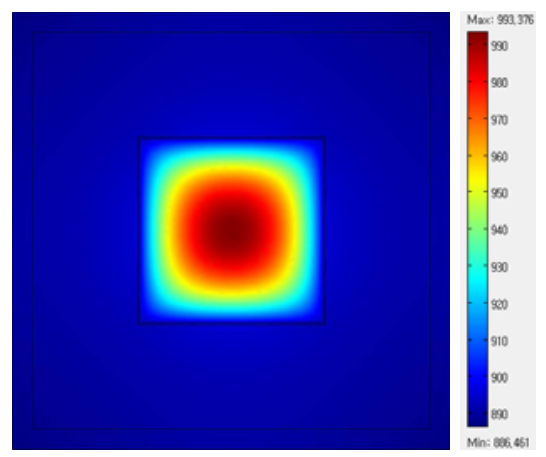

(a) RTPV with cubic source design

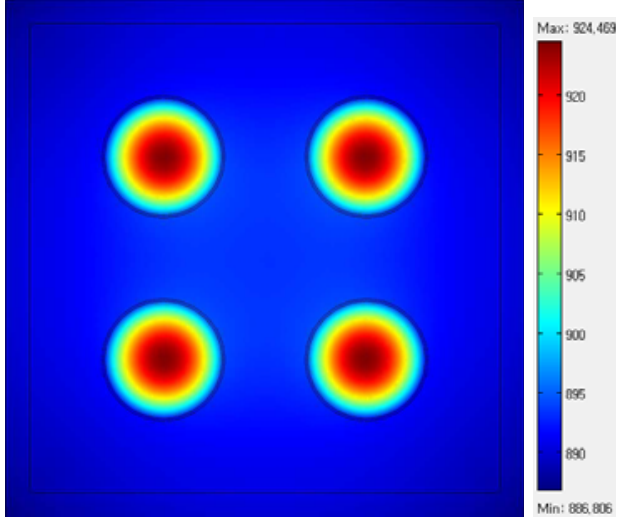

(b) RTPV with four cylindrical sources

Fig. 9. Comparison of temperature distributions at the central planes (with scale in temperature, $\mathrm{K}$ )

Table 4. Maximum and outer face average temperatures for RTPVs having a cubic source and four cylindrical sources (K)

\begin{tabular}{ccc}
\hline Case & Maximum & $\begin{array}{c}\text { Average on } \\
\text { the outer face }\end{array}$ \\
\hline Cubic source & 993.4 & 887.5 \\
\hline Four cylindrical sources & 924.5 & 887.5 \\
\hline
\end{tabular}

\section{Conclusion}

In this work, the radiation shielding analysis was performed for 500W RTPVs having different source designs. In particular, a comparative shielding analysis using two different types of source design (i.e., homogeneous cubic source type and heterogeneous cylindrical sources type) was performed to show their relative performances. The results show that the RTPV device using the heterogeneous cylindrical sources has lower dose values by $5 \sim 11 \%$ depending on the packing factor at the measurement cell than the RTPV device using the cubic source type. In addition, we evaluated and analysed the temperature distributions for these two RTPVs having different source designs. The results of the thermal analysis showed that the RTPV having the separated cylindrical sources has much smaller temperature gradients than the one having a cubic homogeneous source. Therefore, it can be concluded that the RTPV design having separated cylindrical sources can be considered as a candidate for the future RTPV design from view point of the radiation dose and the thermal integrity. 


\section{Acknowledgement}

This work was supported by Space Core Technology Program through the National Research Foundation of Korea (NRF) funded by the Ministry of Science, ICT \& Future Planning (No. 2014M1A3A3A02034818).

\section{References}

1. J. Crepeau, Design of a radioisotope thermophotovoltaic power source by laser driven isotope surrogate assembly, 8 (December 2013)

2. I. C. Gauld, ORIGEN-S: Depletion Module to Calculate Neutron Activation, Actinide Transmutation, Fission Product Generation, And Radiation Source Terms (June 2011)

3. D. E. Pelplow, S. M. Bowman, James E. Horwedel, and J. C. Wagner. Monaco/MAVRIC: Computational Resources for Radiation Protection and Shielding in SCALE, Oak Ridge National Laboratory, MS 6172.

4. D. B. Pelowitz, MCNP6 User's Manual Version 1.0, Manual Rev.0. (May 2013)

5. NASA Ames Research Center, RTG/Science Instrument Radiation Interactions Study for Deep Space Promes, Contract No. 2-5222. (1969)

6. S. J. Cheon, S. G. Hong, Korean Nuclear Society, A Radiation Shielding Analysis for 500W Radioisotope Thermo-Photo-Voltaic(RTPV) System with MCNP6 and MONACO/MAVRIC, Transaction of the Korean Nuclear Society Autumn meeting (Octobor 2015)

7. Oak Ridge National Laboratory, Scale: $A$ Comprehensive Modeling and Simulation Suite for Nuclear Safety Analysis and Design, ORNL/TM2005/39, Version 6.1. (June 2011)

8. COMSOL 3.5a, User's Guide 